\title{
Hypoglycemia Alters Striatal Amino Acid Efflux in Perinatal Rats: An In Vivo Microdialysis Study
}

\author{
Faye S. Silverstein, MDCM, Jennifer Simpson, BA, and Kevin F. Gordon, MD
}

\begin{abstract}
In adult brain, during insulin-induced hypoglycemia, striatal extracellular fluid concentrations of the excitatory amino acids glutamate and aspartate rise markedly (fourfold to tenfold). In this study, we used in vivo microdialysis to determine if insulin-induced hypoglycemia altered striatal amino acid efflux in similar fashion in the immature brain. Microdialysis probes were inserted into the right striatum of rats on postnatal day 7. After a 2-hour recovery period, in each animal a 30-minute baseline sample was obtained. Then insulin $(0.6 \mu / \mathrm{kg}$, intraperitoneal injection) was administered $(n=6)$ and dialysate sampling was continued over the next 210 minutes (terminal blood glucose level $<5 \mathrm{mg} /$ di). Untreated control rats $(n=6)$ were sampled over the same time interval. After pre-column derivatization with o-phthaldialdehyde, dialysate samples were assayed by high-pressure liquid chromatography with electrochemical detection to measure their amino acid content; eight amino acids (glutamate, aspartate, taurine, glutamine, alanine, serine, glycine, and asparagine) were consistently detected. In controls, amino acid efflux did not change over $\mathbf{4}$ hours. In hypoglycemic animals, glutamate efflux increased (peak: $238+85 \%$ of baseline, $p=0.02$, repeated measures analysis of variance [ANOVA] ), glutamine efflux declined (to $44 \pm 5 \%$ of baseline, $p=0.002$, ANOVA), and taurine efflux increased (up to $310 \pm 120 \%$ of baseline; $p<0.06$, ANOVA). In contrast with 9- to 12 -fold increases in aspartate efflux reported in adult striatum, aspartate efflux increased only slightly (to $174 \pm 69 \%$ of baseline; not significant). In immature rodent brain, overall trends in striatal amino acid efflux during hypoglycemia are similar to patterns reported in adult animals; however, there are major differences in the timing, duration, and magnitude of these responses.
\end{abstract}

Silverstein FS, Simpson J, Gordon KE. Hypoglycemia alters striatal amino acid efflux in perinatal rats: an in vivo microdialysis study. Ann Neurol 1990;28:516-521

Recent studies, both in vivo and in vitro, suggest that the excitatory amino acid neurotransmitters glutamate and aspartate contribute to the pathogenesis of hypoglycemia-induced selective neuronal necrosis $[1-6]$. In marure experimental animals, striatal and hippocampal extracellular fluid (ECF) concentrations of aspartate and glutamate rise markedly during hypoglycemia [1, 2], and both deafferentation [4] and treatment with excitatory amino acid antagonists [5] attenuate hypoglycemia-induced neuronal damage. In murine cortical cell cultures deprived of glucose, the extent of neuronal degeneration is reduced by addition of specific glutamate antagonists to the medium [6]. Recent studies demonstrated that the immature brain is susceptible to excitatory amino acid-mediated ("excitotoxic") neuronal damage; the glutamate analogues $N$-methyl-Daspartate and quisqualic acid are potent neurotoxins in postnatal day (PND) 7 rats $[7,8]$. It is thus conceivable that elevated synaptic concentrations of endogenous glutamate or aspartate could be neurotoxic at this developmental stage.

Systemic hypoglycemia is readily induced by injection of insulin and verified by measurement of declining blood glucose concentrations. The immature brain may differ in its response to both insulin and hypoglycemia from the mature brain; the immature brain is often considered relatively resistant to the detrimental effects of hypoglycemia because of more efficient utilization of alternate substrates (fatty acids and lactate) as well as lower metabolic demands [9].

In vivo microdialysis is a new method for sampling the ECF content of specific chemicals of interest in defined brain regions [10]. A microdialysis probe is inserted into a designated brain region, the probe is continuously perfused with a physiological solution, and the concentrations of compounds of interest are measured in dialysate samples. In brain, ECF levels of neurotransmitters are believed to reflect regional syn-
From the Departments of Pediatrics and Neurology, University of Michigan, Ann Arbor, MI.

Received Jan 18, 1990, and in revised form Apr 16. Accepted for publication Apr 22, 1990.
Address correspondence to Dr Silverstein, Room 6028, Kresge II, Box 0570, University of Michigan, Ann Arbor, MI 48109. 
aptic concentrations. Several reports have characterized amino acid efflux in striatum and hippocampus of mature animals, and have delineated characteristic changes in their efflux during hypoglycemia $[1,2]$ and cerebral ischemia $[11,12]$.

We recently developed a method for in vivo striatal microdialysis in PND 7, Sprague-Dawley rats [13]. The goals of this study were to use this method to measure striatal ECF neurotransmitter amino acid efflux in PND 7 rats and to determine the impact of severe hypoglycemia on striatal ECF efflux of these compounds.

\section{Materials and Methods \\ Microdialysis Protocol}

Microdialysis probes were implanted into the striatum of PND 7, Sprague-Dawley rat pups, according to previously described methods [13]. Subsequent histological examination of Nissl-stained, horizontal brain sections was done to verify placement of the probe within the midstriatum; only data from pups with accurate probe placement were evaluated (about two-thirds of animals).

The probes had a concentric design, and 2-mm dialyzing tip (outside diameter, $250 \mu \mathrm{m}$; molecular weight cutoff, 6,000; Spectra-Por, Spectrum Medical, Los Angeles, CA). The microdialysis probe's efficiency of recovery for each amino acid was determined in vitro before insertion and the probe was then glued to a rigid plastic mounting system that could be readily affixed to the skull surface [13].

In each animal, a midline scalp flap was excised under ether anesthesia. One to 2 hours later, pups were reanesthetized and placed in a stereotaxic frame. A cranial flap was excised ( $2.5 \mathrm{~mm}$ lateral, $1 \mathrm{~mm}$ posterior to the bregma) and the probe was lowered and glued into place. The probe tip extended 3 to $5 \mathrm{~mm}$ from the skull surface. Probes were perfused with filtered modified Ringer's solution $(147 \mathrm{mM}$ sodium chloride, $4 \mathrm{mM}$ potassium chloride, $3.4 \mathrm{mM}$ calcium chloride; $\mathrm{pH} 7.2$ ) at $1.5 \mu \mathrm{l} / \mathrm{min}$ using a microinfusion pump (CMA 100, Bioanalytical Systems, Inc, West Lafayette, IN). At the end of each experiment, pups were killed by decapitation. Brains were quickly removed and placed in formalin. Blood glucose levels were measured by Dextrostix (Miles, Elkhart, IN) on blood obtained from the carotid arteries.

Animals were allowed to recover for 2 hours in plastic containers, partially submerged in a warm water bath; the duration of the stabilization period was based on results of earlier studies of catecholamine metabolites. Then collection of samples was initiated; eight sequential 30-minute samples were collected on ice from each animal. Surface body temperature was monitored with an electronic thermometer and was maintained at $35.5^{\circ} \mathrm{C}$. The microdialysis protocol was approved by the University of Michigan Committee on Use and Care of Animals.

To induce hypoglycemia, after baseline sampling, pups received an intraperitoneal injection of regular insulin $(0.6 \mu /$ $\mathrm{kg}$ ). This dose initially produced hyperactivity, and predictably resulted in clinical coma 1 to 2 hours later. The majority of pups survived over the next 3.5 hours while the dialysis sampling continued (20\% mortality in insulin-injected pups during this period). However, no pups treated with the same dose of insulin survived beyond 24 hours.

Sixteen additional animals received the same dose of insulin and were killed by decapitation 0.5 to 4.0 hours later. Blood glucose levels were measured to determine the temporal pattern and severity of hypoglycemia (assayed by Dextrostix and glucometer on blood obtained from carotid arteries). Control blood glucose levels ranged from 100 to 120 $\mathrm{mg} / \mathrm{dl}$. In the first hour after insulin administration, glucose values were typically 30 to $40 \mathrm{mg} / \mathrm{dl}$; at 2 hours after injection, values were $20 \mathrm{mg} / \mathrm{dl}$ or less; and at 3 to 4 hours, values were consistently less than $5 \mathrm{mg} / \mathrm{dl}$. This was the lowest dose of insulin that reproducibly elicited profound hypoglycemia.

\section{Chemical Analysis}

All samples were frozen and were subsequently thawed for analysis by high-performance liquid chromatography with electrochemical detection. A precolumn derivatization procedure was used (modified from [14]). Each sample $(45 \mu \mathrm{l})$ was mixed with $22.5 \mu \mathrm{l}$ of a solution containing $27 \mathrm{mg}$ o-phthaldialdehyde in $2 \mathrm{ml}$ of $100 \mathrm{mM}$ borax with $20 \mu \mathrm{l}$ of mercaptoethanol ( $\mathrm{pH} 9.5$ ) for 30 seconds. The internal standard homoserine $(22.5 \mu \mathrm{l})$ was included with each sample to correct for variations in the efficiency of derivatization. Samples $(80 \mu \mathrm{l})$ were injected onto a $\mathrm{C} 18$ reverse phase column ( $5 \mu$ ODS); the mobile phase was a $0.1 \mathrm{M} \mathrm{NaH}_{2} \mathrm{PO}_{4}(\mathrm{pH}$ 6.5) buffer with $35 \%$ methanol. A glassy carbon electrode connected to an electrochemical detector (Bioanalytical Systems) was used (sensitivity, $20 \mathrm{nAmp}$; electrode potential, $0.7 \mathrm{~V}$ versus silver/silver chloride); this relatively low sensitivity optimized the number of quantifiable peaks and minimized baseline noise. The detection thresholds for the assay were in the picomolar range. External standards containing nine amino acids, in the proportions and concentrations expected in the dialysis samples, were assayed daily. In the dialysis samples, the consistently detected amino acids were glutamate, aspartate, glutamine, glycine, asparagine, taurine, serine, and alanine; gamma-aminobutyric acid (GABA) was detected intermittently and chromatograms often included three or four consistent but unidentified peaks.

\section{Data Analysis}

Efflux values, corrected for efficiency of probe recovery, were expressed in $\mathrm{pmol} / \mathrm{min}$. In each animal, efflux values for each compound were expressed as percent of baseline efflux and group means ( \pm standard error of mean [SEM]) for each sampling period were calculated. Descriptive statistics, analysis of variance (ANOVA) for repeated measures, and Fisher's exact test were performed using a microcomputer-based statistical package (Systat) [15].

\section{Results}

Eight amino acids were consistently detected in striatal dialysate samples (Table). Baseline efflux values in controls ranged from $1.3 \pm 0.2$ (aspartate) to $384 \pm 47$ $\mathrm{pmol} / \mathrm{min}$ (glutamine) and values were similar in controls and experimental animals. To compare these results with values derived from microdialysis studies in adult striatum, the baseline values were also used to calculate ECF amino acid concentrations. In PND 7 
Baseline Striatal ECF Amino Acid Effux (pmollmin) ${ }^{\mathrm{a}}$

\begin{tabular}{lcc}
\hline & \multicolumn{1}{c}{ Control } & Experimental \\
\hline Glutamate & $2.1 \pm 0.5$ & $1.8 \pm 0.5$ \\
Aspartate & $1.3 \pm 0.2$ & $1.8 \pm 0.3$ \\
Taurine & $13.3 \pm 2.5$ & $10.3 \pm 1.2$ \\
Asparagine & $17.5 \pm 3.5$ & $19.8 \pm 5.1$ \\
Glutamine & $384.0 \pm 47.0$ & $453.0 \pm 21.0$ \\
Serine & $75.2 \pm 7.8$ & $73.6 \pm 4.2$ \\
Alanine & $40.2 \pm 4.5$ & $29.2 \pm 3.2$ \\
Glycine & $35.6 \pm 2.4$ & $42.0 \pm 9.8$ \\
\hline
\end{tabular}

${ }^{a}$ Baseline values (mean $\pm S E M$ ) for each amino acid were the same in both groups. GABA was inconsistently detected in both groups.

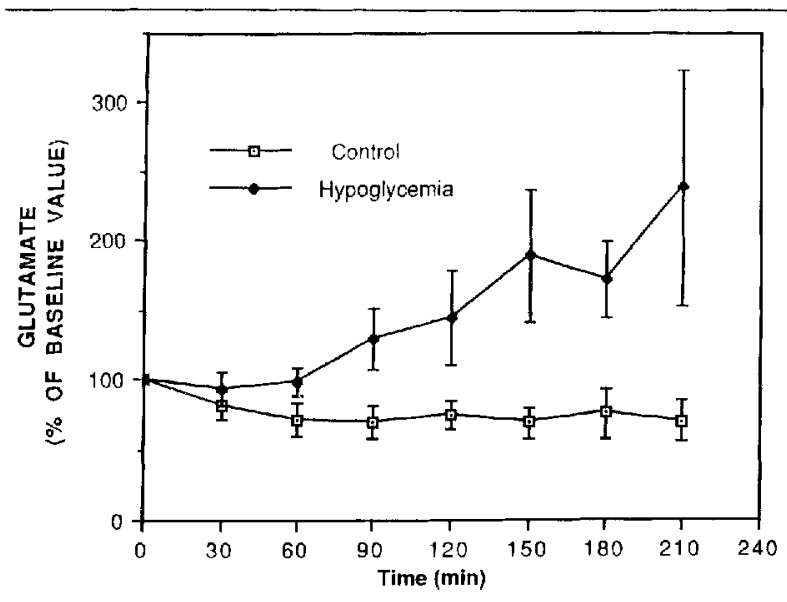

Fig 1. Striatal glutamate effux in control $(n=6)$ and bypoglycemic ( $n=6)$ postnatal day (PND) 7 rats. A microdialysis probe, perfused with modified Ringer's solution at $1.5 \mu \mathrm{ll} / \mathrm{min}$, was inserted into the right striatum; after a 2-bour recovery period, sampling was initiated. Each dialysis sample was collected over 30 minutes. In the experimental group, to induce bypoglycemia, insulin $(0.6 \mu / \mathrm{kg})$ was injected intraperitoneally after the baseline sample was completed (Time "O min"). In each animal, all values were expressed as percent baseline eff $u x$ and group means were calculated. In controls, striatal glutamate efflux declined slightly over time. In bypoglycemic animals, effux gradually increased, peaking at $240 \%$ of baseline in the final sample, repeated measures ANOVA ( $\mathrm{p}=0.02)$ comparing sequential values in experimental and control animals.

rats, the ECF concentration of glutamate $(1.3 \mu \mathrm{M})$ was in the same range as striatal ECF glutamate concentrations reported in adult brain $(0.92 \mu \mathrm{M}[2], 22 \mu \mathrm{M}$ [16]).

In controls, efflux values remained relatively stable over the 4-hour sampling period (Figs 1 through 4). In the experimental group, insulin was injected at the end of the baseline sampling period. In the next two sets of samples, obtained in the first hour after insulin injection (expected blood glucose concentrations, 30 to 40

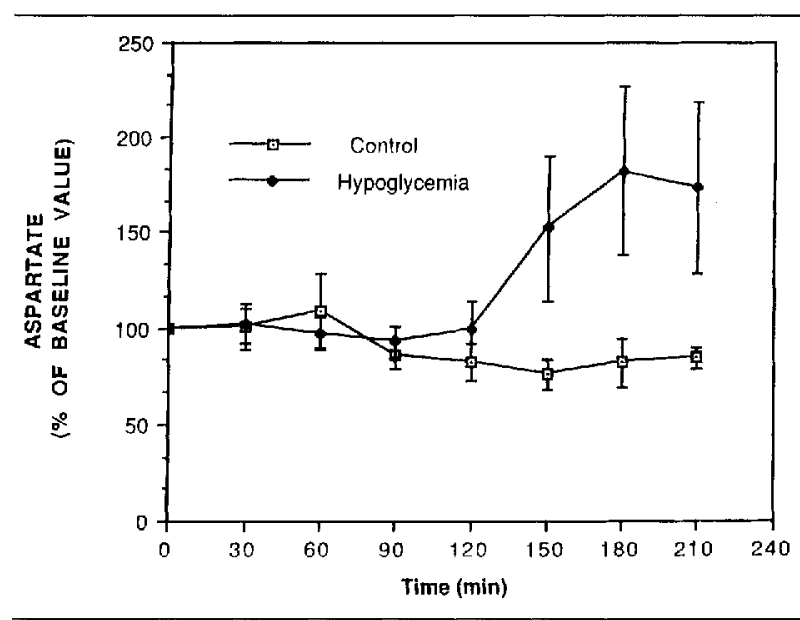

Fig 2. Striatal aspartate efflux in control $(n=6)$ and bypoglycemic $(n=6)$ postnatal day (PND) 7 rats (see Fig 1 legend). Aspartate effux was stable in controls. In bypoglycemic animals, altbough the trend appeared similar to the pattern of glutamate effux (Fig 1), there was considerable variability and values did not differ from those in controls.

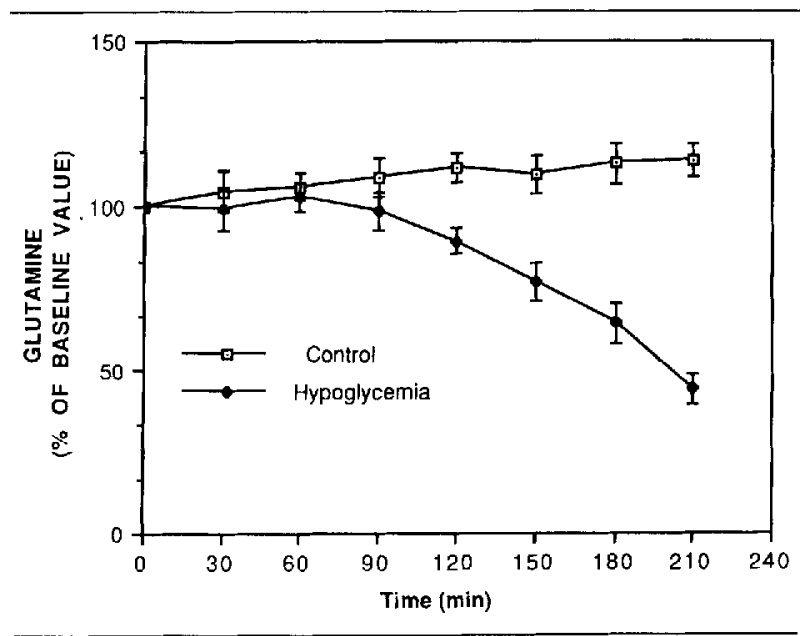

Fig 3. Striatal glutamine effux in control $(n=6)$ and bypoglycemic $(n=6)$ postnatal day (PND) 7 rats (see Fig 1 legend). In the bypoglycemic animals, glutamine efflux progressively declined. In the final sampling period, values fell to less than $50 \%$ of baseline $(\mathrm{p}=0.005)$ repeated measures ANOVA.

$\mathrm{mg} \%$ ), striatal amino acid efflux remained close to baseline values. In the next 2.5 hours, changes in amino acid efflux were detected in hypoglycemic animals.

In controls, striatal glutamate efflux declined slightly over time (see Fig 1). In contrast, in hypoglycemic animals, mean striatal glutamate efflux gradually increased, peaking at $240 \%$ of baseline in the final sample (repeated measures ANOVA, $p=0.02$, comparing sequential values in experimental and control 


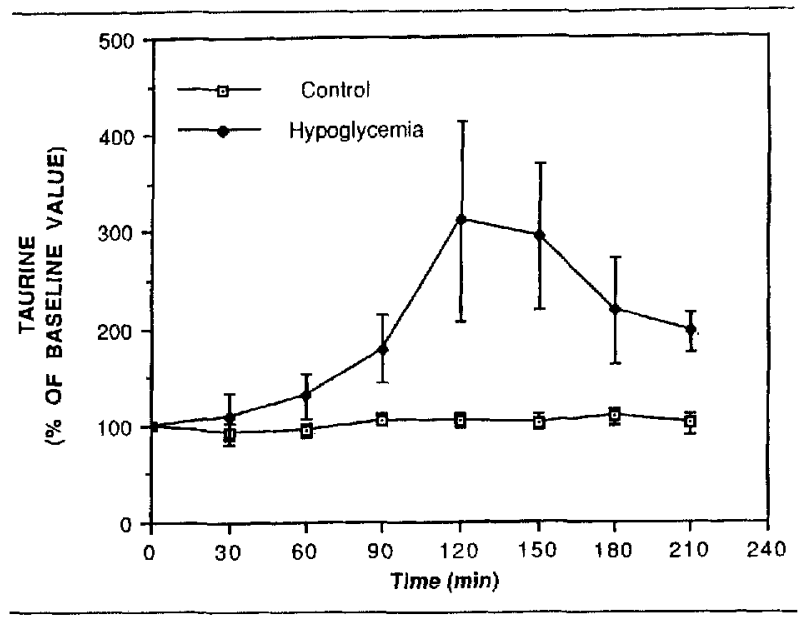

Fig 4. Striatal taurine efflux in control $(n=6)$ and bypoglycemic $(n=6)$ postnatal day (PND) 7 rats (see Fig 1 legend). Efflux values were stable in controls. In the bypoglycemic animals, taurine effux began to rise in the second bour of bypoglycemia; effux values were most consistently elevated during the final sampling period $(196 \pm 20 \%$ of baseline $)(\mathrm{p}=0.058$, repeated measures ANOVA, effect of bypoglycemia over time).

animals). In 5 of 6 hypoglycemic animals, in at least one sampling period glutamate efflux rose to more than $150 \%$ of baseline (versus a peak of the same magnitude in only 1 of 6 controls, $p=0.002$, Fisher's exact test, two-tailed); however, the timing of the peak value varied among animals. Aspartate efflux was also stable in controls; in hypoglycemic animals, although the trend appeared similar to the pattern of glutamate efflux, no significant changes in aspartate efflux were detected (see Fig 2).

In each sample glutamate-aspartate ratios were calculated. There were inter-animal and intra-animal differences in the glutamate-aspartate ratios both at baseline and subsequently in both control and experimental animals but no consistent patterns emerged. In hypoglycemic animals in the baseline samples, the glutamate-aspartate ratio was $0.94 \pm 0.16$ (mean \pm SEM) and in the final sample the corresponding value was $1.2 \pm 0.2$.

Figure 3 compares striatal glutamine efflux in the two groups. In controls, striatal glutamine efflux did not change, whereas in the hypoglycemic animals glutamine efflux progressively declined. Values began to fall in the second hour of hypoglycemia and in the final sampling period fell to less than $50 \%$ of baseline $(p=0.005$, repeated measures ANOVA). Asparagine efflux (data not shown) remained constant until the final sampling period when values declined to 67 $\pm 5 \%$ of baseline.

Striatal taurine efflux was also stable in controls (see Fig 4). In the hypoglycemic animals, values fluctuated. Taurine efflux began to rise in the second hour of hypoglycemia; efflux values were most consistently elevated during the final sampling period (196 $\pm 20 \%$ of baseline) ( $p=0.058$, repeated measures ANOVA, effect of hypoglycemia over time).

A consistent small reduction in striatal glycine efflux was detected in hypoglycemic animals; efflux values declined to $76 \pm 10 \%$ of baseline in the final sampling period ( $p=0.05$, repeated measures ANOVA, effect of hypoglycemia over time). Alanine and serine efflux did not change during hypoglycemia (data not shown). GABA was not consistently detected in either control or hypoglycemic animals.

\section{Discussion}

These results demonstrate that striatal ECF efflux of excitatory amino acid neurotransmitters and related compounds can be monitored in PND 7 rats with in vivo microdialysis. In untreated controls, striatal amino acid efflux is remarkably stable over a 4-hour sampling period and striatal amino acid concentrations are in the same range as values reported in adult brain $[1,2,16]$. In contrast, insulin-induced hypoglycemia markedly altered striatal amino acid efflux.

In hypoglycemic animals glutamate efflux increased gradually; however, peak values were considerably lower than values reported in adult brain (fourfold increase in striatum [3]). It was not feasible to measure blood or striatal ECF glucose levels during the microdialysis experiments. However, based on blood glucose values from additional animals that had received the same dosage of insulin, it appeared that glutamate efflux did not increase until blood glucose values fell to less than $20 \mathrm{mg} \%$. Glutamate efflux increased by at least $50 \%$ over baseline in at least one sampling period in 5 of 6 hypoglycemic animals (versus 1 of 6 controls); however, peak values were not sustained, which could reflect depletion of the releasable glutamate pool. Although the pattern of aspartate efflux during hypoglycemia appeared to parallel the gradual rise in glutamate efflux, values were not significantly different from controls. This finding is in marked contradistinction with responses in adult brain in which 9-fold to 12-fold increases in striatal aspartate concentrations have been reported [3].

Increased glutamate efflux could reflect suppression of re-uptake, increased release, or increased transport into brain; our methods could not distinguish these mechanisms. Neuronal and glial re-uptake are energydependent processes that could fail during hypoglycemia [17]. However, if re-uptake failure accounted for glutamate accumulation during hypoglycemia, sustained elevations in glutamate efflux (rather than a transient peak) and corresponding increases in aspartate efflux, which shares a re-uptake pathway, would be expected. If hypoglycemia stimulated release of glutamate, its source could be a neurotransmitter or a 
metabolic pool. In adult animals, a substantial proportion of striatal glutamate released in response to hypoglycemia appears to be derived from a neurotransmitter pool [3]. However, results of a recent study, done in vitro in a synaptosomal preparation, suggested that with energy depletion a large component of glutamate release was calcium-independent and occurred by reversal of a sodium-coupled uptake carrier [18].

The most consistent change in amino acid efflux we observed was a marked, sustained reduction in glutamine efflux. Glutamine is a precursor for glutamate synthesis [19]. Thus, glutamine depletion may reflect increased glutamate release (together with failure of re-uptake) or its utilization as an alternate carbon group substrate for metabolic pathways [20]. Similar reductions in glutamine efflux have been observed in adult brain during hypoglycemia [2] and also accompanying depolarization-evoked glutamate release [16].

Taurine efflux increased during hypoglycemia, as has been described in adult brain [21]. ECF concentrations of taurine, which may have a role in cerebral osmoregulation [22], also increase during perfusion of glutamate agonists into the adult hippocampus and this stimulation can be blocked by specific glutamate antagonists [23]. As cell swelling is an early component in the neurotoxic effects of glutamate agonists [24], increased taurine release could reflect an adaptive osmoregulatory response.

The developing brain may be less susceptible to hypoglycemia-induced brain injury than is the mature brain. Both lower cerebral metabolic rate (about $10 \%$ of adult values [25]) and greater use of lactate and ketone bodies as alternative substrates to glucose [9] may contribute to this difference. Yet, glucose to some extent remains essential for functioning of the central nervous system and profound insulin-induced hypoglycemia is invariably associated with coma. Of note, doses of insulin which reproducibly elicit neuronal necrosis in striatum of mature animals do not result in similar damage in the immature brain [3] (Silverstein, unpublished observations). We could not determine if the large doses of insulin used in this experiment would result in irreversible neuronal damage, since no animals receiving this dosage survived beyond 24 hours.

The threshold for duration and severity of hypoglycemia that elicits functional derangement and, possibly, irreversible neuronal damage in the developing brain has not been defined. As well, there may be regional and cellular differences in susceptibility to hypoglycemia within the brain. Dialysate amino acid content reflects averages of amino acid efflux from many neuronal populations, as well as glia, within the striatum. If the changes in amino acid efflux we observed coincide with functional disruption of striatal neurons, then the variability in efflux values in hypoglycemic animals may well reflect a wide range of susceptibility to hypoglycemia of specific neuronal populations in the developing striatum.

The effects of inserting a dialysis probe and the limitations of the sampling method must also be considered in interpreting our data. In mature animals insertion of a dialysis probe suppressed blood flow and glucose metabolism maximally in the first 2 hours after insertion [26]. Thus, sampling was initiated 2 hours after the insertions. The stability of efflux values in controls suggests that inserting the probe did not markedly disrupt amino acid efflux. A relatively long sampling period was used in order to maximize the number of detectable compounds; a limitation of this approach is that some biologically important fluctuations in efflux could be obscured.

In clinical practice, the impact of neonatal hypoglycemia on brain development has been difficult to delineate. The risk of adverse neurodevelopmental outcome is increased only in children with concomitant neonatal seizures [27]. It is unclear if the evolution of seizures is a marker for more severe hypoglycemia or an independent pathogenetic factor. We did not directly address the issue of hypoglycemia-induced irreversible brain injury; however, our data suggest that the pathophysiology of severe hypoglycemia in the developing nervous system shares important neurochemical mechanisms with factors that contribute to neuronal damage in the mature brain. Although the extent of stimulation of excitatory amino acid efflux is less than that in mature brain and the timing and duration of altered amino acid efflux elicited by insulin-induced hypoglycemia in perinatal brain vary considerably more than in mature animals, overall, similar changes evolve. Since considerable experimental data indicate that the immature brain is susceptible to the excitotoxic properties of glutamate analogues $[7,8]$, hypoglycemia-induced increases in ECF (and presumably synaptic) concentrations of glutamate may be neurotoxic in the developing brain.

Supported by U.S. Public Health Service grants NS01171 and 26142 (to F. S. S.) and a Centennial fellowship from the Medical Research Council of Canada (to K. G.).

A preliminary report of these data was presented at the annual meeting of the Child Neurology Society, San Antonio, TX, Oct 12, 1989.

\section{References}

1. Sandberg M, Butcher SP, Hagberg M. Extracellular overflow of neuroactive amino acids during severe insulin induced hypoglycemia: in vivo dialysis of the rat hippocampus. J Neurochem $1986 ; 47: 178-184$

2. Butcher SP, Sandberg M, Hagberg $H$, Hamberger A. Cellular 
origins of endogenous amino acids released into the extracellular fluid of the rat striatum during severe insulin-induced hypoglycemia. J Neurochem 1987;47:722-728

3. Auer RN. Progress review: hypoglycemic brain damage. Stroke 1986;17:697-708

4. Wieloch T, Engelsen B, Westerberg E, Auer R. Lesions of the glutamatergic cortico-striatal projections ameliorate hypoglycemic brain damage in the striatum. Neurosci Lett 1985;58:25-30

5. Wieloch $T$. Hypoglycemia induced neuronal damage prevented by an $N$-methyl-D-aspartate antagonist. Science 1985;230:681683

6. Monyer H, Goldberg MP, Choi DW. Glucose deprivation neuronal injury in cortical culture. Brain Res 1989;483:347354

7. Silverstein FS, Chen R, Johnston MV. The glutamate analogue quisqualic acid is neurotoxic in striatum and hippocampus of immature rat brain. Neurosci Lett 1986;71:13-18

8. MCDonald JW, Silverstein FS, Johnston MV. Neurotoxicity of NMDA is markedly enhanced in developing rat central nervous system. Brain Res 1988;459:200-203

9. Cremer JE. Substrate utilization and brain development. J Cereb Blood Flow Metab 1982;2:394-407

10. Ungerstedt U. Measurement of neurotransmitter release by intracranial dialysis. In: Marsden CA, ed. Measurement of neurocransmitter release in vivo. New York: Wiley, 1984:81-107

11. Benveniste H, Drejer J, Schousboe A, Diemer NH. Elevation of the extracellular concentrations of glutamate and aspartate in rat hippocampus during transient cerebral ischemia monitored by intracerebral microdialysis. J Neurochem 1984;43:13691374

12. Hagberg $H$, Lehmann $A$, Sandberg $M$, et al. Ischemia-induced shift of inhibitory and excitatory amino acids from intra- to extracellular compartments. J Cereb Blood Flow Metab 1985; 5:413-419

13. Gordon K, Statman D, Johnston MV, et al. Transient hypoxia alters striatal carecholamine metabolism in immature brain: an in vivo microdialysis study. J Neurochem 1990;54:605-611

14. Donzati BA, Yamamoto BK. An improved and rapid HPLCEC method for the isocratic separation of amino acid neurotransmitters from brain tissue and microdialysis perfusates. Life Sci $1988 ; 43: 913-922$
15. Wilkinson L. Systat: the system for statistics. Evanston, IL: Systat, 1986

16. Young AMJ, Bradford HF. Excitatory amino acid neurotransmitters in the corticostriate pathway: studies using intracerebral microdialysis in vivo. J Neurochem 1986;47:1399-1404

17. Pastuszko A, Wilson DF, Erecinska M. Neurotransmitter metabolism in rat brain synaptosomes. Effect of anoxia and $\mathrm{pH} . \mathrm{J}$ Neurochem 1982;38:1657-1667

18. Kauppinen RA, McMahon HT, Nicholls DG. $\mathrm{Ca}^{++}$dependent and $\mathrm{Ca}^{++}$independent glutamate release, energy status and cytosolic free $\mathrm{Ca}^{++}$concentration in isolated nerve terminals following metabolic inhibition: possible relevance to hypoglycemia and anoxia. Neuroscience 1988;27:175-182

19. Bradford HF, Ward HK, Thomas AJ. Glutamine-a major substrate for nerve endings. J Neurochem 1978;30:1453-1459

20. Norberg K, Siesjo BK. Oxidative metabolism of the cerebral cortex of the rat in severe insulin-induced hypoglycemia. J Neurochem 1976;26:345-352

21. Tossman U, Wieloch T, Ungerstedt $\mathbf{U}$. Gamma-aminobutyric acid and taurine release in the striatum of the rat during hypoglycemic coma studied by microdialysis. Neurosci Lett 1985; $62: 231-235$

22. Lehmann A, Hagberg $\mathrm{H}$, Hamberger $\mathrm{H}$. A role for taurine in the maintenance of homeostasis in the CNS during hyperexcitation? Neurosci Lett 1984;52:34-36

23. Menendez N, Herreras O, Solis JM, et al. Extracellular taurine increase in rat hippocampus evoked by specific glutamate receptor activation is related to the excitatory potency of glutamate agonists. Neurosci Lett 1989;102:64-69

24. Rothman S, Olney JW. Glutamate and the pathophysiology of hypoxic-ischemic beain damage. Ann Neurol 1986;19:105-111

25. Vannucci RC, Christensen MA, Stein DT. Regional cerebral glucose utilization in the immature rat: effect of hypoxiaischemia. Pediatr Res 1989;26:208-214

26. Benveniste $H$, Drejer J, Schousboe A, Diemer NH. Regional cerebral glucose phosphorylation and blood flow after insertion of a microdialysis fiber through the dorsal hippocampus in the rat. J Neurochem 1987;49:729-734

27. Koivisto M, Blanco-Sequeiros M, Krause U. Neonatal symptomatic and asymptomatic hypoglycaemia: a follow-up study. Dev Med Child Neurol 1972;14:603-616 